\title{
Incompressible Viscoelastic Flow of a Generalised Oldroyed-B Fluid through Porous Medium between Two Infinite Parallel Plates in a Rotating System
}

\author{
Dhiman Bose \\ Department of Applied Mathematics \\ University of Calcutta \\ Kolkata 700009 , India
}

\author{
Uma Basu \\ Department of Applied Mathematics \\ University of Calcutta, Kolkata 700009
}

\begin{abstract}
Incompressible viscous fluid flow through a porous medium between two infinite parallel plates with moving upper plate in a rotating system has been studied here. The exact solution of the governing equation for the velocity field has been obtained by using Laplace and finite Fourier sine transformations in series form in terms of Mittage-Leffler function. It can be found that the fluid velocity decreases with the increasing values of fractional calculus parameter $\alpha$ and the permeability of the porous medium $\mathrm{K}$. It can be also observed that the fluid velocity increases with the higher values of the viscosity of the porous medium. The dependence of the velocity field on fractional calculus parameters as well as material parameters has been illustrated graphically.
\end{abstract}

Keywords: Caputo operator; Generalised Oldroyed-B fluid; Laplace transformation: Finite Fourier sine transformation; porous medium.

\section{INTRODUCTION}

In fluid dynamics the study of non-Newtonian fluid flow through porous medium has applications in different fields such as purification of crude oil, petroleum industry, polymer technology, electrostatic precipitation, irrigation, sanitary engineering, food industry etc. The flow behavior of nonNewtonian fluids cannot be described by Newtonian fluid model. For this reason various types of constitutive equations have been proposed and Oldroyed-B fluid model is one of them that has some success in describing non-Newtonian fluids. In recent years fractional calculus approach is found to be quite flexible in describing the viscoelastic fluids. In the approach the time derivative of integer order in the constitutive equation is replaced by Caputo fractional calculus operator. Charyulu and Ram [1] have investigated laminar flow of an incompressible micro polar fluid between two parallel plates with porous lining. Fetecau et al [2] have studied unsteady flow of a second grade fluid between two side walls perpendicular to a plate. Ganapathy [3] have studied oscillatory Couette flow in a rotating system. Jana et al [4] have studied unsteady flow of viscous fluid through a porous medium bounded by a porous plate in a rotating system. Khan et al [5] discussed exact solutions for some oscillating flows of a second grade fluid with a fractional derivative model. Rajagopal [6] investigated unsteady unidirectional flows of a non-Newtonian fluid. Tan et al. [7] discussed exact solution for unsteady couette flow of the generalized second grade fluid. Wenchang et al [8] have studied unsteady flows of viscoelastic fluid with the fractional Maxwell model between two parallel plates.

In present work we have studied the viscoelastic flow of a generalized Oldroyed-B fluid through porous medium between two infinite parallel plates in a rotating system. Here we have used fractional calculus approach in finding exact solution for the velocity field by replacing the time derivative of integer order with Caputo Fractional calculus operator. The exact solutions for the velocity fields are obtained by utilizing the integral transformations in series form in terms of Mittage-Leffler function. We have focused on the behavior of the velocity fields with change in values of porosity parameter and fractional calculus parameters.

\section{CONSTITUTIVE AND GOVERNING EQUATION}

The constitutive relation involving the Cauchy Stress tensor T in a homogeneous and incompressible Oldroyed-B fluid with fractional calculus model can be proposed as

$$
\boldsymbol{T}=-\bar{p} \boldsymbol{I}+\boldsymbol{S}, \boldsymbol{S}+\lambda \frac{D^{\alpha} \boldsymbol{S}}{D t^{\alpha}}=\mu\left(1+\lambda_{r} \frac{D^{\beta}}{D t^{\beta}}\right) \boldsymbol{A}
$$

where $\bar{p}$ is the hydrostatic pressure, $\boldsymbol{I}$ is the identity tensor, $\lambda$ is the time of relaxation, $\lambda_{r}$ is the time of retardation, $\mu$ is the coefficient of viscosity of the fluid, $S$ is the extra stress tensor, $\alpha$ and $\beta$ are the fractional calculus parameters, $V$ is the fluid velocity, $\boldsymbol{A}=\nabla V+(\nabla V)^{T}$ is the Rivlin-Erickson tensor.

$$
\begin{gathered}
\frac{D^{\alpha} \boldsymbol{S}}{D t^{\alpha}}=D_{t}^{\alpha} \boldsymbol{S}+\boldsymbol{V} \cdot \nabla S-(\nabla V) \boldsymbol{S}-\boldsymbol{S}(\nabla V)^{T} \\
\frac{D^{\beta} \boldsymbol{A}}{D t^{\beta}}=D_{t}^{\beta} \boldsymbol{A}+\boldsymbol{V} \cdot \nabla A-(\nabla V) \boldsymbol{A}-\boldsymbol{A}(\nabla V)^{T}
\end{gathered}
$$

$D_{t}^{\alpha}$ and $D_{t}^{\beta}$ are Caputo fractional calculus operators of order $\alpha$ and $\beta$ respectively defined by

$$
D_{t}^{p_{1}} g(t)=\frac{1}{\Gamma\left(1-p_{1}\right)} \int_{0}^{t} g^{\prime}(t)(t-\tau)^{-m} d \tau, 0 \leq p_{1}<1
$$

Where $\Gamma($.$) is the Gamma Function.$

We choose a Cartesian co-ordinate system with $\mathrm{x}$-axis along the lower plate in the direction of the flow, y-axis normal to the plates and $\mathrm{z}$-axis perpendicular to the xy-plane.

We assume the velocity field of the form

$$
\overrightarrow{\boldsymbol{u}}=(u(y, t), 0, w(y, t))
$$

where $u(y, t)$ and $w(y, t)$ are the velocity components in the $\mathrm{x}$ - and $\mathrm{z}$-coordinate directions that are taken along the direction of the parallel plates and normal to the xy-plane respectively. Further we assume the stress of the form $S=S(y, t)$ 
Substituting the Equations (4) and (5) in Equation (1) and taking account the initial condition $S(y, 0)=0,0 \leq y \leq 1$ we get the following Equations

$$
\begin{gathered}
\left(1+\lambda D_{t}^{\alpha}\right) S_{x y}=\mu\left(1+\lambda D_{t}^{\beta}\right) \frac{\partial u(y, t)}{\partial y} \\
\left(1+\lambda D_{t}^{\alpha}\right) S_{y z}=\mu\left(1+\lambda D_{t}^{\beta}\right) \frac{\partial w(y, t)}{\partial y}
\end{gathered}
$$

with $S_{x x}=S_{y y}=S_{z z}=S_{x z}=0, S_{y z}=S_{z y}, S_{x y}=S_{y x}$.

The equations of motion are given by

$$
\begin{gathered}
\rho \frac{\partial u}{\partial t}=\frac{\partial}{\partial y} S_{x y}+2 \rho \Omega w-\frac{\bar{\mu} u}{K} \\
\rho \frac{\partial w}{\partial t}=\frac{\partial}{\partial y} S_{y z}-2 \rho \Omega u-\frac{\bar{\mu} w}{K}
\end{gathered}
$$

where $\bar{\mu}, \rho, \mu, K$ are viscosity of the porous medium, fluid density, viscosity of the fluid and permeability of the porous medium respectively.

Eliminating $S_{x y}$ between the Equations (6) and (8) we get the following governing equation

$$
\begin{array}{r}
\left(1+\lambda D_{t}^{\alpha}\right) \frac{\partial u}{\partial t}=v\left(1+\lambda_{r} D_{t}^{\beta}\right) \frac{\partial^{2} u}{\partial y^{2}} \\
+\left(1+\lambda D_{t}^{\alpha}\right)\left(2 \Omega w-\frac{\bar{\mu} u}{\rho K}\right)
\end{array}
$$

Again eliminating $\mathrm{S}_{\mathrm{yz}}$ between the Equations (7) and (9) we get the following governing equation

$$
\begin{aligned}
&\left(1+\lambda D_{t}^{\alpha}\right) \frac{\partial w}{\partial t}=v\left(1+\lambda_{r} D_{t}^{\beta}\right) \frac{\partial^{2} w}{\partial y^{2}} \\
&-\left(1+\lambda D_{t}^{\alpha}\right)\left(2 \Omega u+\frac{\bar{\mu} w}{\rho K}\right)
\end{aligned}
$$

$v=\frac{\mu}{\rho}$ is the kinematic viscosity.

\section{FORMULATION OF THE PROBLEM}

Let us consider the unsteady flow of a generalized Oldroyed-B fluid through a porous medium bounded by two infinite parallel plates in a rotating system. The plates and the fluid are initially at rest and at $t \rightarrow 0^{+}$the entire system begins to rotate with angular velocity $\Omega$ about the y-axis and at the same time the upper plate moves with constant velocity $U_{0}$ in the $\mathrm{x}$-direction. We take the velocity field of the form $\overrightarrow{\boldsymbol{u}}=(u, 0, w)$ where $\mathrm{u}, \mathrm{w}$ are the velocity components along the $\mathrm{x}$-direction and perpendicular to xy-plane respectively. The governing equations are

$$
\begin{aligned}
&\left(1+\lambda D_{t}^{\alpha}\right) \frac{\partial u}{\partial t}=v\left(1+\lambda_{r} D_{t}^{\beta}\right) \frac{\partial^{2} u}{\partial y^{2}} \\
&+\left(1+\lambda D_{t}^{\alpha}\right)\left(2 \Omega w-\frac{\bar{\mu} u}{\rho K}\right)
\end{aligned}
$$

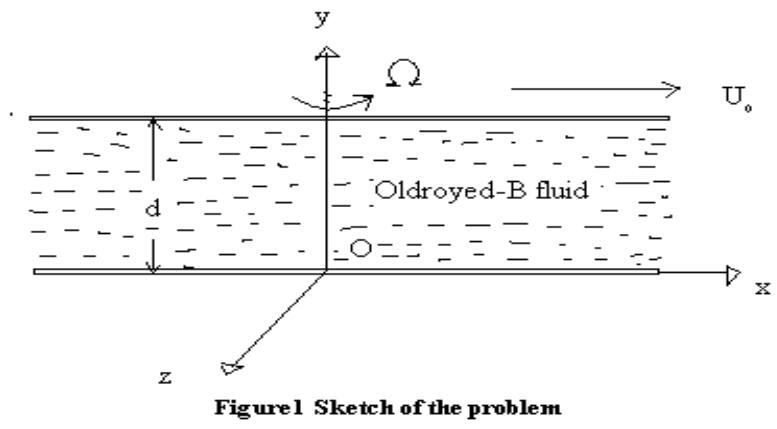

and

$$
\begin{aligned}
&\left(1+\lambda D_{t}^{\alpha}\right) \frac{\partial w}{\partial t}=v\left(1+\lambda_{r} D_{t}^{\beta}\right) \frac{\partial^{2} w}{\partial y^{2}} \\
&-\left(1+\lambda D_{t}^{\alpha}\right)\left(2 \Omega u+\frac{\bar{\mu} w}{\rho K}\right)
\end{aligned}
$$

with the boundary conditions

$$
\begin{array}{r}
u(0, t)=0, w(0, t)=0 \text { for } t>0 \\
\text { and } u(d, t)=U_{0}, w(d, t)=0 \text { for } t>0
\end{array}
$$

and initial conditions

$$
u(y, 0)=0, w(y, 0)=00 \leq y \leq d
$$

Combining the Equations (12) and (13), we have

$$
\begin{gathered}
\left(1+\lambda D_{t}^{\alpha}\right) \frac{\partial q}{\partial t}=v\left(1+\lambda_{r} D_{t}^{\beta}\right) \frac{\partial^{2} q}{\partial y^{2}} \\
-\left(2 i \Omega+\frac{\bar{\mu}}{\rho K}\right)\left(1+\lambda D_{t}^{\alpha}\right) q
\end{gathered}
$$

subject to the boundary and initial conditions

$$
\begin{aligned}
& q(0, t)=0 \text { and } q(d, t)=U_{0} \text { for } \mathrm{t}>0 \\
& q(y, 0)=0,0 \leq y \leq 1
\end{aligned}
$$

We introduce the non-dimensional variables

$$
y^{*}=\frac{y}{d}, q^{*}=\frac{q}{U_{0}}, t^{*}=\frac{t \Omega^{2}}{v}
$$

Then the Equation (16) can be written in terms of nondimensional variables as

$$
\begin{gathered}
\left(1+\lambda^{*} D_{t^{*}}^{\alpha}\right) \frac{\partial q^{*}\left(y^{*}, t^{*}\right)}{\partial t^{*}}=v^{*}\left(1+\lambda_{r}^{*} D_{t^{*}}^{\beta}\right) \frac{\partial^{2} q^{*}\left(y^{*}, t^{*}\right)}{\partial y^{* 2}} \\
-\frac{v}{\Omega^{2}}\left(2 i \Omega+\frac{\bar{\mu}}{\rho K}\right)\left(1+\lambda^{*} D_{t^{*}}^{\alpha}\right) q^{*}\left(y^{*}, t^{*}\right)
\end{gathered}
$$
dimensional variables.

Dropping the asterisk sign we get the dimensionless governing equation as 


$$
\begin{gathered}
\left(1+\lambda D_{t}^{\alpha}\right) \frac{\partial q(y, t)}{\partial t}=v\left(1+\lambda_{r} D_{t}^{\beta}\right) \frac{\partial^{2} q(y, t)}{\partial y^{2}} \\
-M\left(1+\lambda D_{t}^{\alpha}\right) q(y, t)
\end{gathered}
$$

where $M=\frac{v}{\Omega^{2}}\left(2 i \Omega+\frac{\bar{\mu}}{\rho K}\right)$ is complex

constant

The dimensionless boundary and initial conditions are

$$
\begin{gathered}
q(0, t)=0 \text { and } q(1, t)=1 \text { for } t>0 \\
q(y, 0)=0,0 \leq y \leq 1
\end{gathered}
$$

Multiplying both sides of the Equation (21) by $\sin (n \pi y)$ and then integrating with respect to y from 0 to 1 and using the boundary conditions (22a) we get the following equation

$$
\begin{gathered}
\left(1+\lambda D_{t}^{\alpha}\right) \frac{d}{d t} q_{s}(n, t)=v\left(1+\lambda_{r} D_{t}^{\beta}\right)\left[n \pi(-1)^{n+1}\right. \\
\left.-(n \pi)^{2} q_{s}(n, t)\right]-M\left(1+\lambda D_{t}^{\alpha}\right) q_{s}(n, t)
\end{gathered}
$$

where $q_{s}(n, t)$ is the finite Fourier sine transformation of $q(y, t)$ defined by

$$
q_{s}(n, t)=\int_{0}^{1} q(y, t) \sin (n \pi y) d y \quad n=1,2,3, \ldots .
$$

Taking Laplace transformation of both sides of Equation (23) and using $q_{s}(n, 0)=0$ we get

$$
\overline{q_{s}}(n, s)=\frac{v n \pi(-1)^{n+1}}{s\left[(s+M)\left(1+\lambda s^{\alpha}\right)+v(n \pi)^{2}\left(1+\lambda_{r} s^{\beta}\right)\right]}
$$

Where $\overline{q_{s}}(n, s)$ is the Laplace transformation of $q_{s}(n, t)$ defined by $\bar{q}_{s}(n, s)=\int_{0}^{\infty} e^{-s t} q_{s}(n, t) d t$, 's' is the Laplace transform parameter.

In order to avoid the lengthy calculation of contour integration and residues we rewrite the Equation (24) in series form as the following

$$
\begin{aligned}
\bar{q}_{s}(n, s)= & \frac{(-1)^{n+1}}{n \pi}\left[\frac{1}{s}-\sum_{k=0}^{\infty} \frac{(-1)^{k}}{v^{k+1}(n \pi)^{2(k+1)}}\right. \\
& \times \sum_{m=0}^{k+1} \frac{(k+1) !}{m !(k+1-m) !} \frac{\lambda^{m}}{\lambda_{r}^{k+1}} \sum_{j=0}^{k+1} \frac{(k+1) !}{j !(k+1-j) !} M^{j} \\
& \times \frac{s^{\alpha m+k-j}}{\left(s^{\beta}+\lambda_{r}^{-1}\right)^{k+1}}-\sum_{k=0}^{\infty} \frac{(-1)^{k}}{v^{k}(n \pi)^{2 k}} \sum_{m=0}^{k} \frac{k !}{m !(k-m) !} \\
& \left.\times \frac{\lambda^{m}}{\lambda_{r}^{k-1}} \sum_{j=0}^{k} \frac{k !}{j !(k-j) !} M^{j} \frac{s^{\alpha m+\beta-1+k-j}}{\left(s^{\beta}+\lambda_{r}^{-1}\right)^{k+1}}\right]
\end{aligned}
$$

Inserting $M=\frac{v}{\Omega^{2}}\left(2 i \Omega+\frac{\bar{\mu}}{\rho K}\right)$ and using the binomial theorem we get from the Equation (25)

$$
\begin{aligned}
\bar{q}_{s}(n, s)=\frac{(-1)^{n+1}}{n \pi}\left[\frac{1}{s}-\sum_{k=0}^{\infty} \frac{(-1)^{k}}{v^{k+1}(n \pi)^{2(k+1)}}\right. \\
\quad \times \sum_{m=0}^{k+1} \frac{(k+1) !}{m !(k+1-m) !} \frac{\lambda^{m}}{\lambda_{r}^{k+1}} \sum_{j=0}^{k+1} \frac{(k+1) !}{j !(k+1-j) !}
\end{aligned}
$$$$
\times \frac{s^{\alpha m+k-j}}{\left(s^{\beta}+\lambda_{r}^{-1}\right)^{k+1}} \frac{v^{j}}{\Omega^{2 j}} \sum_{l=0}^{j} \frac{j !}{l !(j-l) !}\left(\frac{\bar{\mu}}{\rho K}\right)^{j-l}
$$

$$
\times\left(\cos \frac{l \pi}{2}+i \sin \frac{l \pi}{2}\right)
$$

$$
-\sum_{k=0}^{\infty} \frac{(-1)^{k}}{v^{k}(n \pi)^{2 k}} \sum_{m=0}^{k} \frac{k !}{m !(k-m) !} \frac{\lambda^{m}}{\lambda_{r}^{k-1}} \sum_{j=0}^{k} \frac{k !}{j !(k-j) !}
$$$$
\times \frac{s^{\alpha m+\beta-1+k-j}}{\left(s^{\beta}+\lambda_{r}^{-1}\right)^{k+1}} \frac{v^{j}}{\Omega^{2 j}} \sum_{l=0}^{j} \frac{j !}{l !(j-l) !}\left(\frac{\bar{\mu}}{\rho K}\right)^{j-l}
$$

$$
\left.\times\left(\cos \frac{l \pi}{2}+i \sin \frac{l \pi}{2}\right)\right]
$$

Now we have an important Laplace transformation of the Mittage-Leffler function

$$
\int_{0}^{\infty} e^{-s t} t^{\alpha n+\lambda-1} E_{\alpha, \lambda}^{(n)}\left(-a t^{\alpha}\right) d t=\frac{n ! s^{\alpha-\lambda}}{\left(s^{\alpha}+a\right)^{n+1}}
$$

Where $E_{\alpha, \lambda}(z)$ is the Mittage-Leffler function

$$
E_{\alpha, \lambda}^{(n)}(z)=\frac{d^{n}}{d z^{n}} E_{\alpha, \lambda}(z)=\sum_{j=0}^{\infty} \frac{(j+n) ! z^{j}}{j ! \Gamma(\alpha j+\alpha n+\lambda)}
$$

Taking inverse Laplace transformation we get from the Equation(26)

$$
\begin{aligned}
& q_{s}(n, t)=\frac{(-1)^{n+1}}{n \pi}-\frac{(-1)^{n+1}}{n \pi} \sum_{k=0}^{\infty} \frac{(-1)^{k}}{v^{k+1}(n \pi)^{2(k+1)}} \\
& \quad \times \sum_{m=0}^{k+1} \frac{(k+1) !}{m !(k+1-m) !} \frac{\lambda^{m}}{\lambda_{r}^{k+1}} \sum_{j=0}^{k+1} \frac{(k+1) !}{j !(k+1-j) !} \\
& \times \frac{v^{j}}{\Omega^{2 j}} \sum_{l=0}^{j} \frac{j !}{l !(j-l) !}\left(\frac{\bar{\mu}}{\rho K}\right)^{j-l}(2 \Omega)^{l} \frac{t^{\beta(k+1)-\alpha m-k+j-1}}{k !} \\
& \quad \times E_{\beta, \beta-\alpha m-k+j}^{(k)}\left(-\lambda_{r}^{-1} t^{\beta}\right)\left(\cos \frac{l \pi}{2}+i \sin \frac{l \pi}{2}\right) \\
& \quad \frac{(-1)^{n+1} \sum_{j=0}^{\infty} \frac{(-1)^{k}}{n \pi} \sum_{k=0}^{k} \frac{k}{v^{k}(n \pi)^{2 k}} \sum_{m=0}^{k !(k-j) !} \frac{k !}{m !(k-m) !} \frac{\lambda^{m}}{\lambda_{r}^{k-1}}}{\Omega^{2 j} \sum_{l=0}^{j} \frac{j !}{l !(j-l) !}\left(\frac{\bar{\mu}}{\rho K}\right)^{j-l}(2 \Omega)^{l}}
\end{aligned}
$$




$$
\begin{aligned}
& \times \frac{t^{\beta k-\alpha m-k+j}}{k !} E_{\beta, 1-\alpha m-k+j}^{(k)}\left(-\lambda_{r}^{-1} t^{\beta}\right) \\
& \times\left(\cos \frac{l \pi}{2}+i \sin \frac{l \pi}{2}\right)
\end{aligned}
$$

Taking inverse Fourier sine transformation of the Equation (29) and comparing the real and imaginary parts of both sides of the resulting equation we get

$$
\begin{aligned}
u(y, t)= & y+2 \sum_{n=1}^{\infty} \frac{(-1)^{n}}{n \pi} \sin (n \pi y) \sum_{k=0}^{\infty} \frac{(-1)^{k}}{v^{k+1}(n \pi)^{2(k+1)}} \\
& \times \sum_{m=0}^{k+1} \frac{(k+1) !}{m !(k+1-m) !} \frac{\lambda^{m}}{\lambda_{r}^{k+1}} \sum_{j=0}^{k+1} \frac{(k+1) !}{j !(k+1-j) !} \\
& \times \frac{v^{j}}{\Omega^{2 j}} \sum_{l=0}^{j} \frac{j !}{l !(j-l) !}\left(\frac{\bar{\mu}}{\rho K}\right)^{j-l}(2 \Omega)^{l} \frac{t^{\beta(k+1)-\alpha m-k+j-1}}{k !} \\
+ & \quad \sum_{n=1}^{\infty} \frac{(-1)^{n}}{n \pi} \sin (n \pi y) \sum_{\beta, \beta-\alpha m-k+j}^{(k)} \frac{(-1)^{k}}{v^{k}(n \pi)^{2 k}} \sum_{m=0}^{k} \frac{k !}{m !(k-m) !} t^{\beta} \cos \frac{l \pi}{2} \\
& \times \frac{\lambda^{m}}{\lambda_{r}^{k-1}} \sum_{j=0}^{k} \frac{k !}{j !(k-j) !} \frac{v^{j}}{\Omega^{2 j}} \sum_{l=0}^{j} \frac{j !(j-l) !}{l !}\left(\frac{\bar{\mu}}{\rho K}\right)^{j-l}(2 \Omega)^{l} \\
& \times \frac{t^{\beta k-\alpha m-k+j}}{k !} E_{\beta, 1-\alpha m-k+j}^{(k)}\left(-\lambda_{r}^{-1} t^{\beta}\right) \cos \frac{l \pi}{2} \quad(30)
\end{aligned}
$$$$
w(y, t)=2 \sum_{n=1}^{\infty} \frac{(-1)^{n}}{n \pi} \sin (n \pi y) \sum_{k=0}^{\infty} \frac{(-1)^{k}}{v^{k+1}(n \pi)^{2(k+1)}}
$$$$
\times \sum_{m=0}^{k+1} \frac{(k+1) !}{m !(k+1-m) !} \frac{\lambda^{m}}{\lambda_{r}^{k+1}} \sum_{j=0}^{k+1} \frac{(k+1) !}{j !(k+1-j) !}
$$$$
\times \frac{v^{j}}{\Omega^{2 j}} \sum_{l=0}^{j} \frac{j !}{l !(j-l) !}\left(\frac{\bar{\mu}}{\rho K}\right)^{j-l}(2 \Omega)^{l} \frac{t^{\beta(k+1)-\alpha m-k+j-1}}{k !}
$$$$
\times E_{\beta, \beta-\alpha m-k+j}^{(k)}\left(-\lambda_{r}^{-1} t^{\beta}\right) \sin \frac{l \pi}{2}
$$$$
+2 \sum_{n=1}^{\infty} \frac{(-1)^{n}}{n \pi} \sin (n \pi y) \sum_{k=0}^{\infty} \frac{(-1)^{k}}{v^{k}(n \pi)^{2 k}} \sum_{m=0}^{k} \frac{k !}{m !(k-m) !}
$$$$
\times \frac{\lambda^{m}}{\lambda_{r}^{k-1}} \sum_{j=0}^{k} \frac{k !}{j !(k-j) !} \frac{v^{j}}{\Omega^{2 j}} \sum_{l=0}^{j} \frac{j !}{l !(j-l) !}\left(\frac{\bar{\mu}}{\rho K}\right)^{j-l}
$$$$
\times \frac{t^{\beta k-\alpha m-k+j}}{k !} E_{\beta, 1-\alpha m-k+j}^{(k)}\left(-\lambda_{r}^{-1} t^{\beta}\right) \sin \frac{l \pi}{2}
$$

The non-dimensional shear stresses at the stationary plate $(y=0)$ due to the primary and secondary flows is given by

$\tau_{x}+i \tau_{y}=\left(\frac{\partial q}{\partial y}\right)_{y=0}$

$$
=1+2 \sum_{n=1}^{\infty}(-1)^{n} \sum_{k=0}^{\infty} \frac{(-1)^{k}}{v^{k+1}(n \pi)^{2(k+1)}}
$$$$
\times \sum_{m=0}^{k+1} \frac{(k+1) !}{m !(k+1-m) !} \frac{\lambda^{m}}{\lambda_{r}^{k+1}} \sum_{j=0}^{k+1} \frac{(k+1) !}{j !(k+1-j) !}
$$$$
\times \frac{v^{j}}{\Omega^{2 j}} \sum_{l=0}^{j} \frac{j !}{l !(j-l) !}\left(\frac{\bar{\mu}}{\rho K}\right)^{j-l}(2 \Omega)^{l} \frac{t^{\beta(k+1)-\alpha m-k+j-1}}{k !}
$$$$
\times E_{\beta, \beta-\alpha m-k+j}^{(k)}\left(-\lambda_{r}^{-1} t^{\beta}\right)\left(\cos \frac{l \pi}{2}+i \sin \frac{l \pi}{2}\right)
$$$$
+2 \sum_{n=1}^{\infty}(-1)^{n} \sum_{k=0}^{\infty} \frac{(-1)^{k}}{v^{k}(n \pi)^{2 k}} \sum_{m=0}^{k} \frac{k !}{m !(k-m) !}
$$$$
\times \frac{\lambda^{m}}{\lambda_{r}^{k-1}} \sum_{j=0}^{k} \frac{k !}{j !(k-j) !} \frac{v^{j}}{\Omega^{2 j}} \sum_{l=0}^{j} \frac{j !}{l !(j-l) !}\left(\frac{\bar{\mu}}{\rho K}\right)^{j-l}
$$

$$
\begin{gathered}
\times \frac{t^{\beta k-\alpha m-k+j}}{k !} E_{\beta, 1-\alpha m-k+j}^{(k)}\left(-\lambda_{r}^{-1} t^{\beta}\right) \\
\times\left(\cos \frac{l \pi}{2}+i \sin \frac{l \pi}{2}\right)
\end{gathered}
$$

Separating the real and imaginary parts of both sides of the Equation (32) we get the shear stress components due to the primary and secondary flows at the stationary plate $y=0$ as

$$
\begin{aligned}
\tau_{x}=1+2 \sum_{n=1}^{\infty}(-1)^{n} \sum_{k=0}^{\infty} \frac{(-1)^{k}}{v^{k+1}(n \pi)^{2(k+1)}} \\
\quad \times \sum_{m=0}^{k+1} \frac{(k+1) !}{m !(k+1-m) !} \frac{\lambda^{m}}{\lambda_{r}^{k+1}} \sum_{j=0}^{k+1} \frac{(k+1) !}{j !(k+1-j) !}
\end{aligned}
$$$$
\times \frac{v^{j}}{\Omega^{2 j}} \sum_{l=0}^{j} \frac{j !}{l !(j-l) !}\left(\frac{\bar{\mu}}{\rho K}\right)^{j-l}(2 \Omega)^{l} \frac{t^{\beta(k+1)-\alpha m-k+j-1}}{k !}
$$$$
\times E_{\beta, \beta-\alpha m-k+j}^{(k)}\left(-\lambda_{r}^{-1} t^{\beta}\right) \cos \frac{l \pi}{2}
$$$$
+2 \sum_{n=1}^{\infty}(-1)^{n} \sum_{k=0}^{\infty} \frac{(-1)^{k}}{v^{k}(n \pi)^{2 k}} \sum_{m=0}^{k} \frac{k !}{m !(k-m) !}
$$$$
\times \frac{\lambda^{m}}{\lambda_{r}^{k-1}} \sum_{j=0}^{k} \frac{k !}{j !(k-j) !} \frac{v^{j}}{\Omega^{2 j}} \sum_{l=0}^{j} \frac{j !}{l !(j-l) !}\left(\frac{\bar{\mu}}{\rho K}\right)^{j-l}
$$ 


$$
\begin{aligned}
& \times \frac{t^{\beta k-\alpha m-k+j}}{k !} E_{\beta, 1-\alpha m-k+j}^{(k)}\left(-\lambda_{r}^{-1} t^{\beta}\right) \cos \frac{l \pi}{2} \\
& \tau_{y}=2 \sum_{n=1}^{\infty}(-1)^{n} \sum_{k=0}^{\infty} \frac{(-1)^{k}}{v^{k+1}(n \pi)^{2(k+1)}} \\
& \times \sum_{m=0}^{k+1} \frac{(k+1) !}{m !(k+1-m) !} \frac{\lambda^{m}}{\lambda_{r}^{k+1}} \sum_{j=0}^{k+1} \frac{(k+1) !}{j !(k+1-j) !} \\
& \times \frac{v^{j}}{\Omega^{2 j}} \sum_{l=0}^{j} \frac{j !}{l !(j-l) !}\left(\frac{\bar{\mu}}{\rho K}\right)^{j-l}(2 \Omega)^{l} \frac{t^{\beta(k+1)-\alpha m-k+j-1}}{k !} \\
& \times E_{\beta, \beta-\alpha m-k+j}^{(k)}\left(-\lambda_{r}^{-1} t^{\beta}\right) \sin \frac{l \pi}{2} \\
& +2 \sum_{n=1}^{\infty}(-1)^{n} \sum_{k=0}^{\infty} \frac{(-1)^{k}}{v^{k}(n \pi)^{2 k}} \sum_{m=0}^{k} \frac{k !}{m !(k-m) !} \\
& \times \frac{\lambda^{m}}{\lambda_{r}^{k-1}} \sum_{j=0}^{k} \frac{k !}{j !(k-j) !} \frac{v^{j}}{\Omega^{2 j}} \sum_{l=0}^{j} \frac{j !}{l !(j-l) !}\left(\frac{\bar{\mu}}{\rho K}\right)^{j-l} \\
& \times \frac{t^{\beta k-\alpha m-k+j}}{k !} E_{\beta, 1-\alpha m-k+j}^{(k)}\left(-\lambda_{r}^{-1} t^{\beta}\right) \sin \frac{l \pi}{2}
\end{aligned}
$$

\section{RESULTS AND DISCUSSION}

Figure 2 depicts the behavior of the primary velocity component $\mathrm{u}$ against the distance from the lower plate measured along y-axis for different values of the fractional calculus parameter $\alpha$. From the figure it is observed that as $\alpha$ takes higher values the flow velocity decreases and the profile of the velocity curve changes from parabolic slope. Figure 3 depicts the primary velocity for three values of the rotational parameter $\Omega$. The flow velocity u decreases with increase in $\Omega$ and this is similar to the case in Figure 2. Figure 4 explains that the flow velocity $u$ decreases with the increasing values of permeability parameter $K$ that is porosity produces a resistance force in the flow field. The nature of flow patterns are slightly deviated from the parabolic type. Figure 5 depicts the velocity component $u$ against the distance from the lower plate measured along $y$-axis for different values of the fractional calculus parameter $\beta$. As $\beta$ increases the flow velocity $\mathrm{u}$ also increases and the nature of the velocity curves are more parabolic with increase in $\beta$. It is observed from the Figure 6 that the flow velocity $u$ increases with the increase in the parameter of viscosity $\mu$. The velocity field $\mathrm{u}$ is plotted in Figure 7 against distance from the lower plate at different time t. It is noted from the figure that the velocity field $u$ decreases and the nature of flow pattern are less parabolic with increase in time t. Figure 8 depicts the velocity field $u$ against $y$ for different values of the kinematic viscosity $v$. The flow velocity, $u$ increases with the increase in $v$. It is observed from the Figure 9 that the secondary velocity w decreases with increase in the parameter $\alpha$ and the parabolic nature of the flow pattern is not effected by $\alpha$. The influence of the parameter $\beta$ on the secondary flow velocity $w$ is illustrated in Figure 10. It is seen from the figure that ' $w$ ' increases with the increase in $\beta$ but the parabolic nature of the flow pattern remains fixed. Figure 11 depicts the shear stress $\tau_{x}$ at the stationary plate due to primary flow ' $u$ ' against the kinematic viscosity $v$ for different values of the permeability parameter
$K$ of the porous medium. It is evident from the figure that shear stress $\tau_{x}$ decreases with increase in $K$. It is observed from the Figure 12 that as $\alpha$ takes higher values the shear stress $\tau_{x}$ decreases. Figure 13 shows the dependence of $\tau_{x}$ on the fractional calculus parameter $\beta$. As ' $\beta$ ' takes higher values, the shear stress $\tau_{x}$ increases. Figure 14 depicts the shear stress $\tau_{y}$ against kinematic viscosity $\mathrm{v}$ at the stationary plate due to the secondary flow for different values of $\alpha$. It is seen from the figure that $\tau_{y}$ decreases with the increase in $\alpha$. Figure 15 reveals that the shear stress $\tau_{y}$ increases with the increase in the fractional calculus parameter $\beta$.

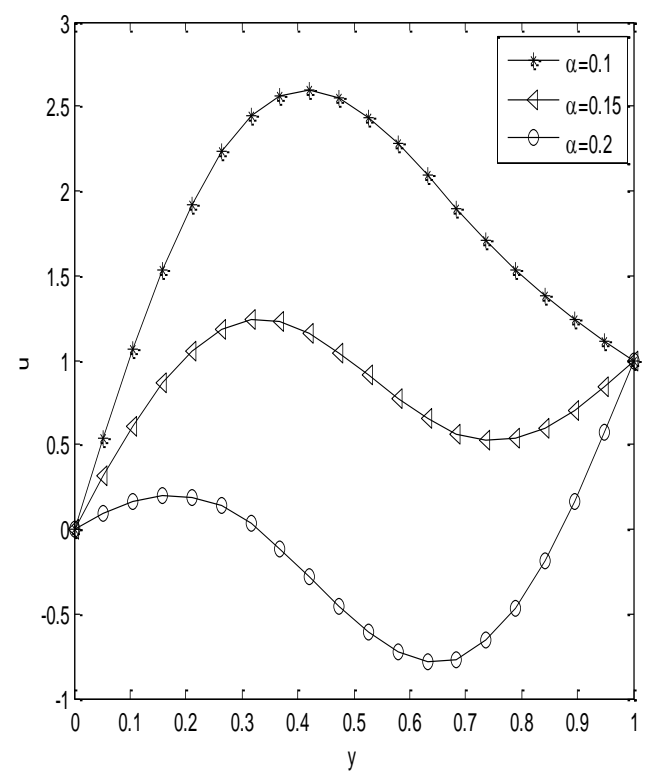

Figure 2 :The velocity field $u$ is depicted against the distance from the lower plate for different values of the fractional calculus parameter $\alpha . v=0.02, \lambda=2, \lambda_{r}=$ $3, \Omega=0.2, \mu=0.4, \beta=0.8, t=1, K=0.6, \rho=0.1$

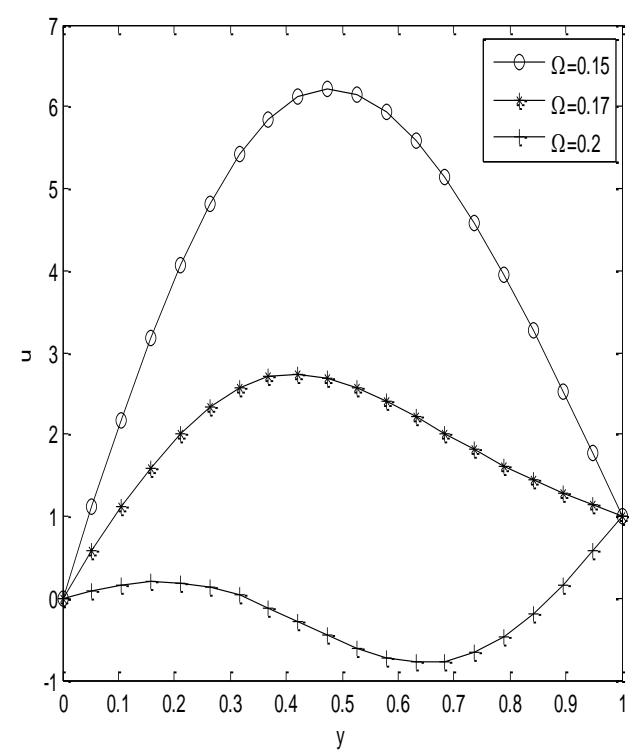

Figure 3: The velocity field $u$ is depicted against the distance from the lower plate for different values of $\Omega$. $v=0.02, \lambda=2, \lambda_{r}=3, \mu=0.4, \alpha=0.2, \beta=$ $0.8, \mathrm{t}=1, \mathrm{~K}=0.6, \rho=0.1$ 


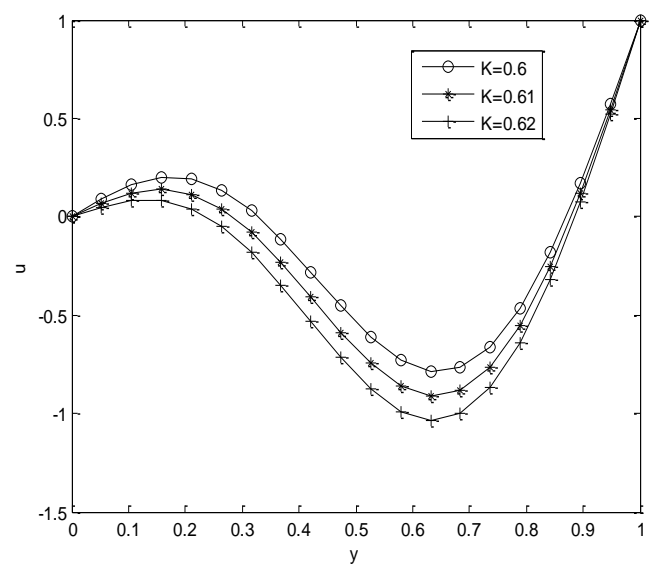

Figure 4: The velocity field $u$ is depicted against the distance from the lower plate for different values of parameter of permeability $K . \quad v=0.02, \lambda=2, \lambda_{r}=$ $3, \Omega=0.2, \mu=0.4, \alpha=0.2, \beta=0.8, t=1, \rho=0.1$

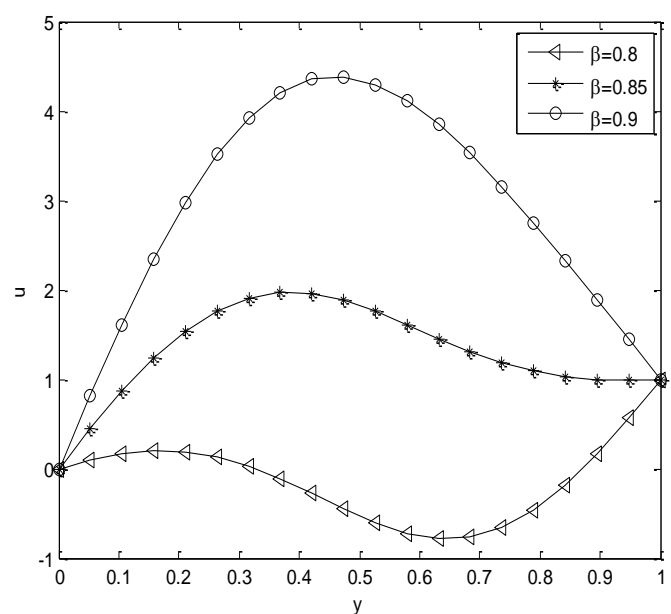

Figure 5: The velocity field $u$ is depicted against the distance from the lower plate for different values of fractional calculus parameter $\beta . \quad v=0.02, \lambda=2, \lambda_{\mathrm{r}}=$ $3, \Omega=0.2, \mu=0.4, \alpha=0.2, t=1, K=0.6, \rho=0.1$

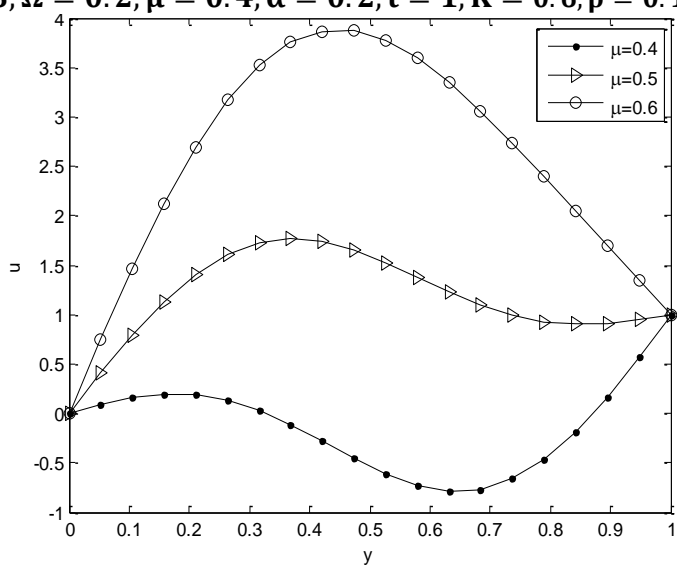

Figure 6: The velocity field $u$ is depicted against the distance from the lower plate for different values of viscosity parameter $\mu . \quad v=0.02, \lambda=2, \lambda_{r}=3, \Omega=$ $0.2, \alpha=0.2, \beta=0.8, t=1, K=0.6, \rho=0.1$

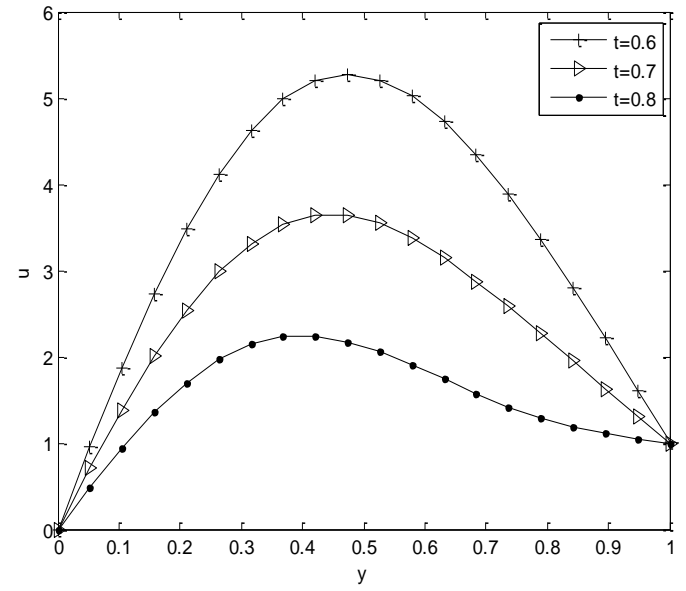

Figure 7: The velocity field $u$ is depicted against the distance from the lower plate at different time t. $\quad v=$ $0.02, \lambda=2, \lambda_{r}=3, \Omega=0.2, \mu=0.4, \alpha=0.2, \beta=$ $0.8, K=0.6, \rho=0.1$

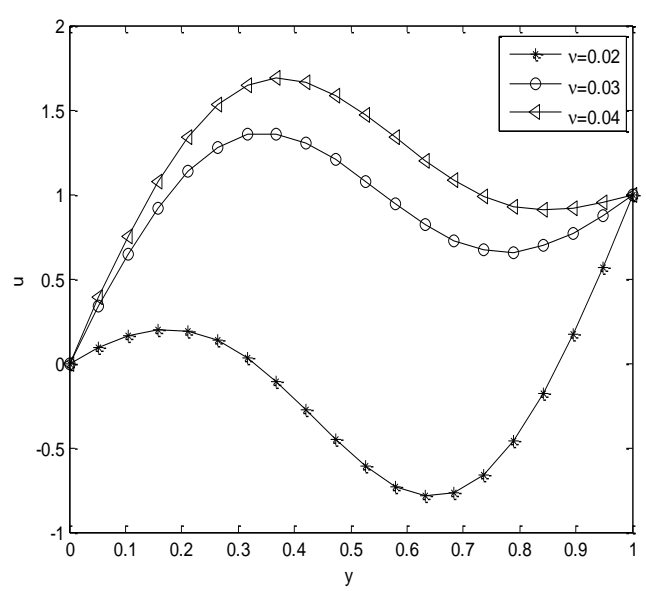

Figure 8: The velocity field $u$ is depicted against the distance from the lower plate for different values of material parameter $v . \lambda=2, \lambda_{r}=3, \Omega=0.2, \mu=$

$0.4, \alpha=0.2, \beta=0.8, t=1, K=0.6, \rho=0.1$

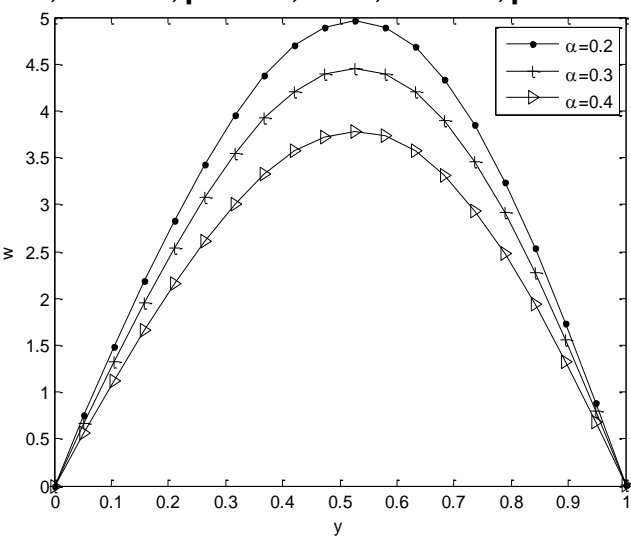

Figure 9: The velocity field $w$ is depicted against the distance from the lower plate for different values of fractional calculus parameter $\alpha . v=0.02, \lambda=2, \lambda_{\mathrm{r}}=$ $3, \Omega=0.2, \mu=0.4, \beta=0.8, t=1, K=0.6, \rho=0$. 


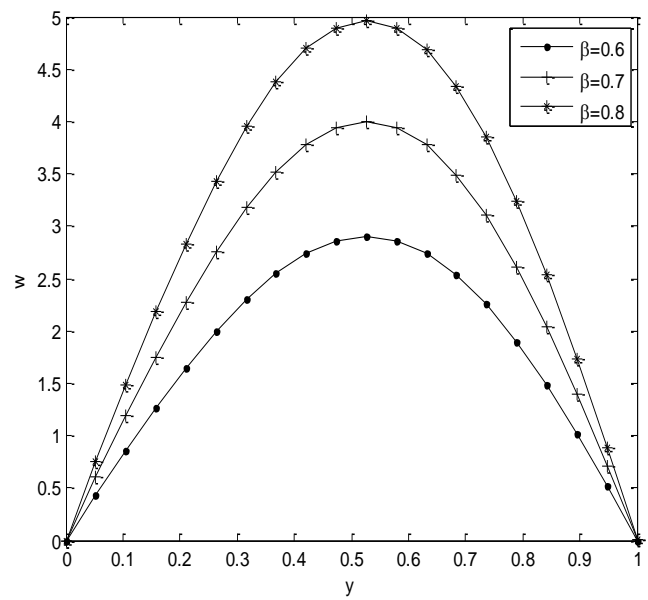

Figure 10: The velocity field $w$ is depicted against the distance from the lower plate for different values of fractional calculus parameter $\beta . \quad v=0.02, \lambda=2, \lambda_{\mathrm{r}}=$ $3, \Omega=0.2, \mu=0.4, \alpha=0.2, t=1, K=0.6, \rho=0.1$

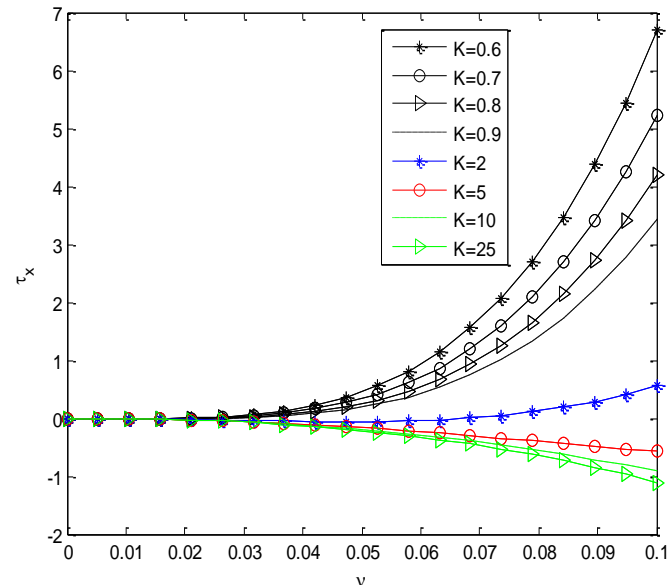

Figure 11: The shear stress $\tau_{x}$ at the stationary plate due to the primary flow is depicted against the kinematic viscosity $v$ for different values of permeability of porous medium K. $\lambda=2, \lambda_{r}=3, \Omega=0.2, \mu=0.4, \alpha=0.2, \beta=$ $0.8, t=1, \rho=0.1$

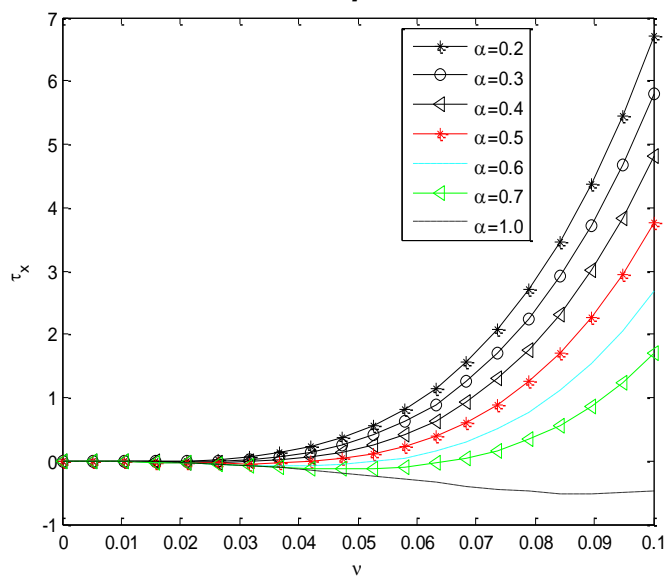

Figure 12: The shear stress $\tau_{x}$ at the stationary plate due to the primary flow is depicted against the kinematic viscosity $v$ for different values of parameter $\alpha . \quad \lambda=$ $2, \lambda_{r}=3, \Omega=0.2, \mu=0.4, \beta=0.8, t=1, K=0.6, \rho=$ 0.1

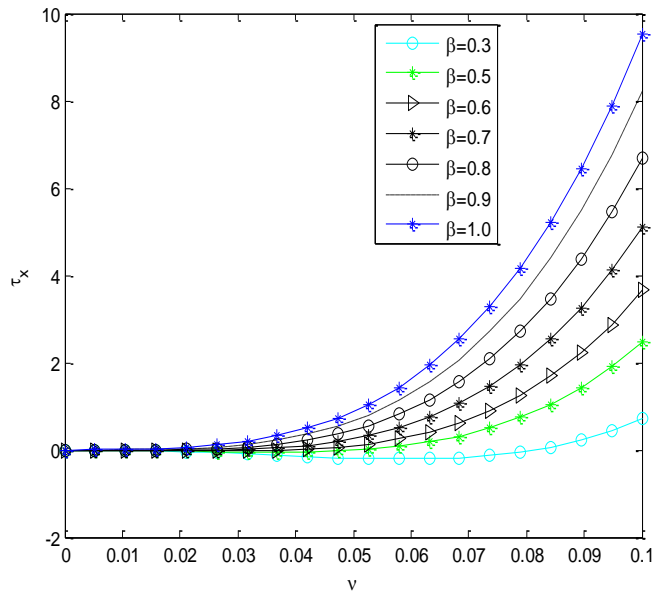

Figure 13: The shear stress $\tau_{x}$ at the stationary plate due to the primary flow is depicted against the kinematic viscosity $v$ for different values of parameter $\beta . \lambda=2, \lambda_{r}=$ $3, \Omega=0.2, \mu=0.4, \alpha=0.2, t=1, K=0.6, \rho=0.1$

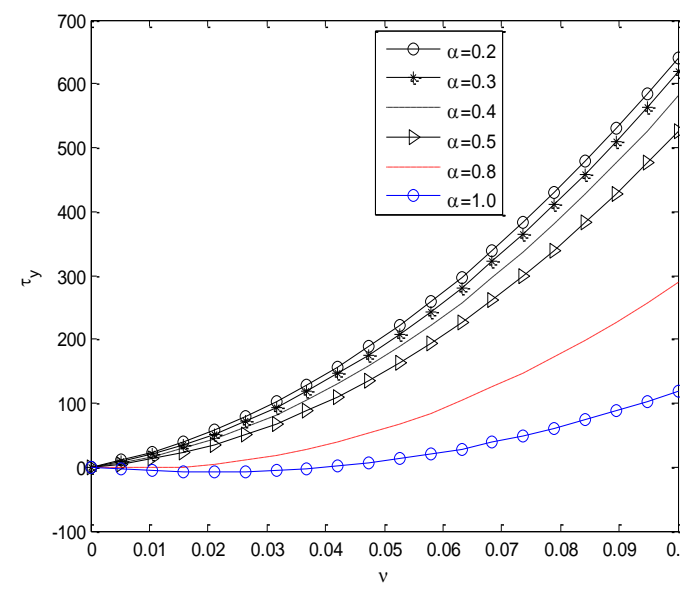

Figure 14: The shear stress $\tau_{y}$ at the stationary plate due to the secondary flow is depicted against the kinematic viscosity $v$ for different values of parameter $\alpha . \lambda=2, \lambda_{r}=$ $3, \Omega=0.2, \mu=0.4, \beta=0.8, t=1, K=0.6, \rho=0.1$

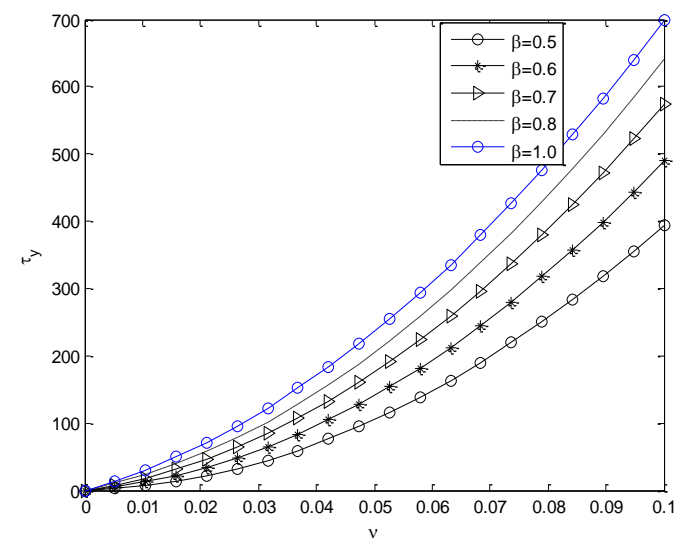

Figure 15: The shear stress $\tau_{y}$ at the stationary plate due to the secondary flow is depicted against the kinematic viscosity $\mathbf{v}$ for different values of parameter $\beta$.. $\lambda=$ $2, \lambda_{r}=3, \Omega=0.2, \mu=0.4, \alpha=0.2, t=1, K=0.6, \rho=$ 0.1

\section{CONCLUSION}

The flow of an incompressible generalised Oldroyed-B fluid through a porous medium between two infinite parallel plates 
in a rotating system is considered. The exact solution for the velocity field is obtained by utilizing Laplace and finite Fourier sine transformation in series forms in terms of Mittage-Leffler function. The influence of the fractional calculus parameters as well as material parameters on the velocity field has been illustrated graphically. Moreover the effects of permeability parameter $K$ of the porous medium, fractional calculus parameters $\alpha$ and $\beta$ on the shear stresses $\tau_{x}$ and $\tau_{y}$ due to the primary and secondary velocity components respectively have been discussed graphically.

\section{ACKNOWLEDGEMENTS}

The authors wish to thank the reviewers for useful comments which have lead to the improvement of our work in the present form.

\section{REFERENCES}

[1] Charyulu, V.N. and Ram, M.S. 2010. Laminar flow of an incompressible micro polar fluid between two parallel plates with porous lining. Int. J. Applied Math and Mech.6, 81-92.

[2] Fetecau, C., Hayat, T., Fetecau, C. and Ali, N. 2008. Unsteady flow of a second grade fluid between two side walls perpendicular to a plate. Nonlinear Anal: Real World Appl. 9, 1236-1252.
[3] Ganapathy, R. 1994. A note on oscillatory Couette Flow in a rotating system. Journal of Applied Mechanics. 61, 208-209.

[4] Jana, M., Maji, S.L.,Das, S.and Jana, R.N. 2010 Unsteady flow of viscous fluid through a porous medium bounded by a porous plate in a rotating system. Journal of Porous Media.13, 645-653.

[5] Khan, M., Ali, S.H and Qi, H. 2009. Exact solutions for some oscillating flows of a second grade fluid with a fractional derivative model. Mathematical and Computer Modelling. 49, 1519-1530.

[6] Rajagopal, K.R.1982. A note on unsteady unidirectional flows of a non-Newtonian fluid. International Journal of Non-Linear Mechanics. 17, 369-373.

[7] Tan, W.C., Xian, F. and Wei, L. 2002. Exact solution for the unsteady Couette flow of the generalized second grade fluid. China Sci. Bull. 47,1226-1228.

[8] Wenchang,T.,Wenxiao, P. and Mingyu, X. 2003. A note on unsteady flows of a viscoelastic fluid with the fractional Maxwell model between two parallel plates. International Journal of Non-Linear Mechanics. 38, 645650. 